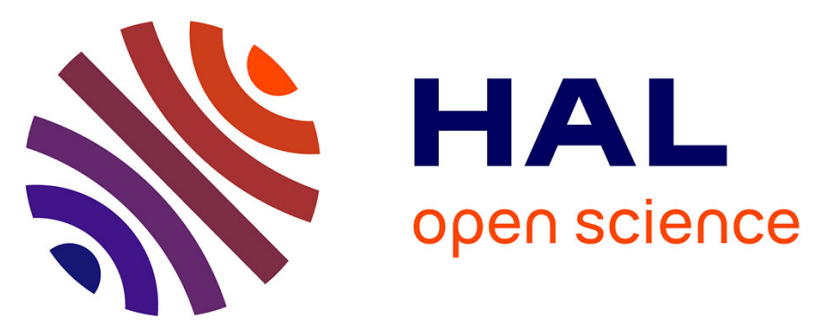

\title{
Evolutionary transition to XY sex chromosomes associated with Y-linked duplication of a male hormone gene in a terrestrial isopod
}

Aubrie Russell, Sevarin Borrelli, Rose Fontana, Joseph Laricchiuta, Jane Pascar, Thomas Becking, Isabelle Giraud, Richard Cordaux, Christopher H Chandler

\section{To cite this version:}

Aubrie Russell, Sevarin Borrelli, Rose Fontana, Joseph Laricchiuta, Jane Pascar, et al.. Evolutionary transition to XY sex chromosomes associated with Y-linked duplication of a male hormone gene in a terrestrial isopod. Heredity, 2021, 127 (3), pp.266-277. 10.1038/s41437-021-00457-2 . hal-03376536

\author{
HAL Id: hal-03376536 \\ https://hal.science/hal-03376536
}

Submitted on 15 Oct 2021

HAL is a multi-disciplinary open access archive for the deposit and dissemination of scientific research documents, whether they are published or not. The documents may come from teaching and research institutions in France or abroad, or from public or private research centers.
L'archive ouverte pluridisciplinaire HAL, est destinée au dépôt et à la diffusion de documents scientifiques de niveau recherche, publiés ou non, émanant des établissements d'enseignement et de recherche français ou étrangers, des laboratoires publics ou privés. 
1 Evolutionary transition to $\mathrm{XY}$ sex chromosomes associated with $\mathrm{Y}$-linked duplication of a

2 male hormone gene in a terrestrial isopod

3

4 Aubrie Russell ${ }^{1}$, Sevarin Borrelli ${ }^{1}$, Rose Fontana ${ }^{1}$, Joseph Laricchiuta ${ }^{1}$, Jane Pascar ${ }^{1}, 2$, Thomas

$5 \quad$ Becking $^{3}$, Isabelle Giraud ${ }^{3}$, Richard Cordaux ${ }^{3}$, Christopher H. Chandler ${ }^{1 *}$

6

7 'Department of Biological Sciences, State University of New York at Oswego, Oswego, NY,

8 United States

$9 \quad{ }^{2}$ Current address: Biology Department, Syracuse University, Syracuse, NY, United States

$10{ }^{3}$ Laboratoire Ecologie et Biologie des Interactions, Equipe Ecologie Evolution Symbiose,

11 Université de Poitiers, UMR CNRS 7267 Poitiers, France

12

$13{ }^{*}$ Corresponding author:

14 Christopher H. Chandler

15 Department of Biological Sciences

16 State University of New York at Oswego

17 christopher.chandler@oswego.edu

18

19

Key words: Wolbachia, Oniscidea, Trachelipus, androgenic gland hormone, AGH 


\section{Abstract}

22

23 Sex chromosomes are highly variable in some taxonomic groups, but the evolutionary mechanisms underlying this diversity are not well understood. In terrestrial isopod crustaceans, evolutionary turnovers in sex chromosomes are frequent, possibly caused by Wolbachia, a vertically-transmitted endosymbiont causing male-to-female sex reversal. Here, we use surgical manipulations and genetic crosses, plus genome sequencing, to examine sex chromosomes in the terrestrial isopod Trachelipus rathkei. Although an earlier cytogenetics study suggested a ZZ/ZW sex chromosome system in this species, we surprisingly find multiple lines of evidence that in our study population, sex is determined by an $X X / X Y$ system. Consistent with a recent evolutionary origin for this $X X / X Y$ system, the putative male-specific region of the genome is small. The genome shows evidence of Y-linked duplications of the gene encoding the androgenic gland hormone, a major component of male sexual differentiation in isopods. Our analyses also uncover sequences horizontally acquired from past Wolbachia infections, consistent with the hypothesis that Wolbachia may have interfered with the evolution of sex determination in $T$. rathkei. Overall, these results provide evidence for the co-occurrence of multiple sex chromosome systems within $T$. rathkei, further highlighting the relevance of terrestrial isopods as models for the study of sex chromosome evolution. 


\section{Introduction}

Although sexual reproduction is shared by most eukaryotes, a variety of different cues can trigger individuals to follow a male, female, or hermaphroditic developmental plan (Conover and Kynard, 1981; Tingley and Anderson, 1986; Janzen and Phillips, 2006; Ospina-Álvarez and Piferrer, 2008; Verhulst et al., 2010). In many eukaryotes, sex is primarily determined genotypically, often involving sex chromosomes, although other mechanisms, such as polygenic systems and haplodiploidy, are also known (Vandeputte et al., 2007; Heimpel and de Boer, 2008). Sex chromosomes in animals are usually grouped into two main classes: $X Y$ systems, in which males are heterogametic $(X Y)$ and females are homogametic $(X X)$; and $Z W$ systems, in which females are heterogametic (ZW) and males are homogametic (ZZ). However, non-genetic cues can also play an important role in some species. For instance, environmental factors, such as temperature or population density, influence or determine phenotypic sex in reptiles, fishes, and invertebrates (Conover and Kynard, 1981; Tingley and Anderson, 1986; Janzen and Phillips, 2006). In some cases, cytoplasmic factors, including sex-reversing endosymbionts, such as Wolbachia, microsporidia, and paramyxids can serve as a sex-determining signal (Terry et al., 1998; Bouchon et al., 1998; Kageyama et al., 2002; Negri I et al., 2006; Pickup and Ironside, 2018).

Evolutionary theory holds that the formation of sex chromosomes begins when an autosome acquires a sex-determining locus (Rice, 1996). Subsequently, recombination around the sexdetermining locus is selected against because of sexually antagonistic selection (Bergero and Charlesworth, 2009). For instance, selection should favor mutations that are beneficial in males but deleterious in females when those alleles are linked to a dominant male-determining allele; recombination, on the other hand, would break up this linkage and result in females that carry these male-beneficial alleles. The non-recombining region is then expected to spread in the 
presence of continued sexually antagonistic selection, and may eventually span the whole sex chromosome, except for the usual presence of a small recombining pseudo-autosomal region (Charlesworth et al., 2005). Once recombination has ceased, the non-recombining sex chromosome, such as the $\mathrm{Y}$ chromosome in mammals or the $\mathrm{W}$ chromosome in birds, is expected to degenerate. Non-recombining genes frequently undergo pseudogenization, acquiring nonsense mutations or transposable element insertions (Charlesworth and Charlesworth, 2000). At the same time, gene trafficking can occur when selection promotes the translocation of formerly autosomal genes to the sex chromosomes (Emerson et al., 2004).

Different species appear to be at different stages of sex chromosome evolution. For instance, the sex chromosomes of therian mammals are highly conserved, having originated $\sim 160$ million years ago (Potrzebowski et al., 2008; Veyrunes et al., 2008). The highly degenerated, heteromorphic $\mathrm{Y}$ chromosome represents an advanced stage of sex chromosome evolution. In other taxonomic groups, on the other hand, sex chromosomes appear to undergo more frequent evolutionary turnovers (Ross et al., 2009; Cioffi et al., 2013; Vicoso and Bachtrog, 2015; Myosho et al., 2015; Pennell et al., 2018; Jeffries et al., 2018). Such young sex chromosomes may have little or no recombination suppression, differentiation in gene content, or sex chromosome dosage compensation, and may not be detectable by traditional cytogenetic methods because they are visually indistinguishable (homomorphic) (Gamble et al., 2014; Vicoso and Bachtrog, 2015). Sex chromosomes may even be polymorphic within a species, with different sex-determining loci segregating within or among populations (Orzack et al., 1980; Traut, 1994; Ogata et al., 2008; Meisel et al., 2016).

Unfortunately, we still have a limited understanding of why evolutionary turnovers of sex chromosomes are rare in some groups but frequent in others. A variety of models have been proposed to explain why these turnovers occur, including sexual antagonism, where a novel 
sex-determining allele spreads because of its association with another allele with sex-specific effects (van Doorn and Kirkpatrick 2007); the accumulation of deleterious mutations on the nonrecombining sex chromosome (Blaser et_al._2013); and the 'hot potato' model, which suggests that the accumulation of both sexually antagonistic and deleterious mutations can lead to repeated sex chromosome turnovers (Blaser et_al._2014). In some organisms, interactions with vertically transmitted reproductive endosymbionts are also thought to influence the evolution of their hosts' sex determination mechanisms (Rigaud et al., 1997; Cordaux et al., 2011). However, many of these models have been difficult to test in nature. This problem is exacerbated by the fact that, while sex chromosomes have been extensively studied in model organisms like Drosophila, studies are more sparse in non-model organisms.

One group that has received relatively little attention is crustaceans. Different crustacean species show a variety of distinct sex determining mechanisms, yet there are very few crustacean species in which candidate master sex-determining genes have been identified (Chandler et al., 2017, 2018). Within crustaceans, perhaps one of the best-studied groups in terms of sex determination is the terrestrial isopods (Oniscidea). Terrestrial isopod species have a mix of $X Y$ and $Z W$ systems, along with reports of a few parthenogenic species and populations (Fussey, 1984; Johnson, 1986; Rigaud et al., 1997). The bacterial endosymbiont Wolbachia also influences sex determination by causing male-to-female sex reversal in some isopod hosts (Bouchon et al., 1998; Cordaux et al., 2004). In fact, interactions with Wolbachia are thought to drive rapid evolutionary turnover of the sex chromosomes in terrestrial isopods. For instance, in the common pillbug Armadillidium vulgare, a copy of the Wolbachia genome horizontally integrated into the host genome (known as the $f$ element) led to the origin of a new W chromosome (Leclercq et al., 2016). A recent phylogenetic analysis also identified several transitions in heterogametic systems along the isopod phylogeny, including closely related species pairs with different sex chromosome systems (Becking et al., 2017). Moreover, only a 
few species of terrestrial isopods are known to have heteromorphic sex chromosomes, in which the $\mathrm{X}$ and $\mathrm{Y}$, or $\mathrm{Z}$ and $\mathrm{W}$, chromosomes are distinguishable in cytogenetics experiments (Rigaud et al., 1997), and WW or YY individuals are often viable and fertile (Juchault and Rigaud, 1995; Becking et al., 2019), suggesting that the $W$ and $Y$ chromosomes have not lost any essential genes in these species.

In this study, we examined sex determination in the widespread species Trachelipus rathkei. This species was previously established by cytogenetic methods to have heteromorphic, albeit slightly, Z and W sex chromosomes (Mittal and Pahwa, 1980), and is nested within a clade that appears ancestrally to possess a ZZ/ZW sex determination mechanism (Becking êt $\underline{\text { al }} \underline{-}_{2} \underline{2}$ 2017). We sought to confirm female heterogamety by crossing females to sex-reversed males (which have female genotypes but male phenotypes), and assessing the sex ratio of the resulting progenies, which will differ depending on the sex chromosome system (XX neo-male $\times X X$ female yields all XX and therefore $100 \%$ female offspring; ZW neo-male $\times Z W$ female expected to produce $1 / 4 \mathrm{ZZ}, 1 / 2 \mathrm{ZW}$, and $1 / 4 \mathrm{WW}$ offspring, thus $75 \%$ female or $66.7 \%$ female depending on whether WW genotypes are viable). Surprisingly, we found that, at least in our focal population, sex is determined by an $\mathrm{XX} / \mathrm{XY}$ system, suggesting a recent sex chromosome turnover. To test this hypothesis, we performed whole-genome sequencing. Consistent with a recent origin of an $X X / X Y$ sex determination system, we find evidence that the putative malespecific region is small relative to the whole genome, and we identified a male-specific, partial duplication of the androgenic gland hormone (AGH) gene, a rare example of a candidate sexdetermining gene in a crustacean. In addition, although our study population does not appear to harbor current Wolbachia infections, we find genomic evidence of past infections. Overall, our results are consistent with the hypothesis that Wolbachia endosymbionts may have interfered with the evolution of sex determination in T. rathkei. 


\section{$\underline{\text { Animal collection and husbandry }}$}

We sampled wild isopods from Rice Creek Field Station (RCFS) at SUNY Oswego in Oswego, NY. We captured animals using a combination of methods. First, we haphazardly searched through leaf litter, logs, and rocks. We also used "potato traps", made by carving out a 1-2 cm diameter core from a potato and placing it in the litter for 1-2 weeks. Finally, we constructed pitfall traps from plastic cups buried in the ground with the rim of the cup flush with the ground. The primary species captured were Oniscus asellus and T. rathkei, but we also captured Philoscia muscorum, Hyloniscus riparius, Trichoniscus pusillus, and occasionally Cylisticus convexus. Species identification was performed in the field and confirmed in the lab, where we also determined the phenotypic sex of specimens.

Isopods were housed in plastic food storage containers with holes in the lids for air exchange, on a substrate of moistened soil. Containers were checked twice weekly. Animals were fed carrots and dried leaves ad libitum. The photoperiod was kept on a schedule of 18:6 light hours: dark hours in the summer and 14:10 in the winter. We isolated ovigerous females in individual containers, and separated offspring from their mothers upon emergence from the marsupium. We initially sexed offspring at six to eight weeks old, and separated males from females to prevent sibling mating. We then double-checked offspring sex at roughly two week intervals thereafter until four months of age to watch for individuals that might have shown late signs of sexual differentiation. Terrestrial isopods are known to store sperm from a single mating to fertilize future broods. Therefore, for experimental crosses we only used $T$. rathkei females that were born in the lab, separated from brothers as soon as they could be sexed, and which had not produced any offspring by 12 months of age. 


\section{$172 \quad$ Wolbachia testing}

We used PCR assays to test for Wolbachia presence in T. rathkei individuals. DNA was extracted from one or two legs, depending on the size of the animal. We ruptured the leg tissue in $400 \mu \mathrm{L}$ deionized water along with a few $0.5 \mathrm{~mm}$ zirconia/silica beads (enough to cover the bottom of the tube) using a bead beater machine. Samples were lysed following a protocol of 2500 RPM for 10 seconds, followed by 4200 RPM for 10 seconds, and finally 4800 RPM for 10 seconds. The tube was then visually inspected to confirm the leg was sufficiently pulverized. We then transferred the lysate to a new tube, added $60 \mu \mathrm{L}$ of a $5 \%$ Chelex® 100 molecular biology grade resin suspension, and incubated for 15 minutes at $100^{\circ}$

C. After incubation, we centrifuged the extract at $16,000 \mathrm{~g}$ for 3 minutes, and reserved $80 \mu \mathrm{L}$ of supernatant for PCR testing. We confirmed successful DNA extraction using the mitochondrial primers HCO2198/LCO1490 (Folmer et al., 1994). We performed PCRs in $10 \mu \mathrm{L}$ reactions, using 4.95 $\mu \mathrm{L}$ of molecular biology grade water, $2 \mu \mathrm{L}$ NEB OneTaq Buffer, $1 \mu \mathrm{L}$ of mixed dNTPS at a final concentration of $2 \mathrm{mM}$ for each dNTP, $1 \mu \mathrm{L}$ of a $5 \mu \mathrm{M}$ solution of each primer, and $0.05 \mu \mathrm{L}$ of NEB OneTaq. For the mitochondrial primer set, PCR conditions included an initial denaturation of $94^{\circ} \mathrm{C}$ for 1 minute; 5 cycles of $94^{\circ} \mathrm{C}$ denaturation for $30 \mathrm{~s}, 45^{\circ} \mathrm{C}$ annealing for $90 \mathrm{~s}$, and $68^{\circ} \mathrm{C}$ extension for $60 \mathrm{~s}$. The samples then underwent 35 cycles of $94^{\circ} \mathrm{C}$ for $30 \mathrm{~s}, 51^{\circ} \mathrm{C}$ for $90 \mathrm{~s}$, and $68^{\circ} \mathrm{C}$ for $60 \mathrm{~s}$. This was followed by a final extension step of $68^{\circ} \mathrm{C}$ for 5 minutes. To test for Wolbachia, we performed PCR using Wolbachia-specific primers targeting the wsp (81f/691r) and ftsZ (ftsZf1/ftsZr1) genes (Werren John H. et al., 1995; Braig et al., 1998). We performed PCRs in $10 \mu \mathrm{L}$ reactions, using $4.95 \mu \mathrm{L}$ of molecular biology grade water, $2 \mu \mathrm{L}$ NEB OneTaq Buffer, $1 \mu \mathrm{L}$ of mixed dNTPs at a final concentration of $2 \mathrm{mM}$ for each dNTP, $1 \mu \mathrm{L}$ of either wsp or ftsZ primers, and $0.05 \mu \mathrm{L}$ of NEB OneTaq. PCR conditions contained an initial denaturation of $95^{\circ} \mathrm{C}$ for 5 minutes, followed by 36 cycles of $95^{\circ} \mathrm{C}$ for $60 \mathrm{~s}, 54^{\circ} \mathrm{C}$ for $60 \mathrm{~s}$, and $68^{\circ} \mathrm{C}$ for 3 
minutes. This was followed by a final extension step at $68^{\circ} \mathrm{C}$ for 10 minutes. Positive PCR tests would not necessarily be able to distinguish between a true infection and a copy of the Wolbachia genome horizontally integrated into the host genome, but the absence of a PCR product should be a reliable indicator that these Wolbachia sequences are not present (at least at detectable levels).

\section{Androgenic gland implantation and crosses}

To test whether sex is determined by a ZZ/ZW or XX/XY system of sex determination in our population of $T$. rathkei, we performed crosses between females and experimentally sexreversed neo-males. Juvenile female $T$. rathkei were implanted with live androgenic glands, according to (Becking et al., 2017). Male donors and female recipients were selected from large lab-reared broods with even ( 1:1) sex ratios. An adult male was sacrificed by decapitation, and live androgenic glands were dissected into Ringer solution (393 mM NaCl, 2 mM KCl, 2 mM $\mathrm{CaCl}_{2} \cdot 2 \mathrm{H}_{2} \mathrm{O}, 2 \mathrm{mM} \mathrm{NaHCO}$ ). Female recipients were between 5 and 8 weeks old, an age at which males and females begin to become distinguishable by the appearance of external male genitalia, but at which sexual development is not complete. Occasionally, young males may still be mistaken for females at this stage, if the male genitalia are not sufficiently developed, and thus it is common for some fraction of the recipients in these experiments to carry male genotypes (Becking et al $_{2}$ 2017); nevertheless, it is important for transplant recipients to be as young as possible for sex reversal to be complete. The androgenic gland was injected using a pulled glass pipette into a hole pierced with a dissecting needle in the 6th or 7 th segment of the juvenile female's pereon. Recipients were isolated in a small plastic container with a moist paper towel for recovery and observation. Experimental animals were monitored for signs of male development. Any surviving animal that failed to develop male genitalia by 4 months postimplantation was considered to be a failed injection. After maturation, adult neo-males were 
placed in individual containers with 1-3 previously unmated females. Crosses were monitored twice weekly to check for signs of reproduction in females. Gravid females were then isolated into their own containers until parturition.

\section{Genome sequencing}

We performed whole-genome sequencing using a combination of Illumina, PacBio, and Oxford Nanopore sequencing, with multiple sequencing samples of each sex (Supplementary Table 1). Because we expected the $T$. rathkei genome to be large, repetitive, and highly polymorphic, and because we expected to need to isolate DNA from multiple individuals, we established a partially inbred laboratory line using offspring from a single female collected from RCFS. We mated brothers and sisters from this female for two generations in the lab prior to collecting genetic samples from the third generation for sequencing. DNA was collected for sequencing using the Qiagen DNEasy Blood and Tissue Kit following the manufacturer's instructions. DNA was quantified using the Qubit DNA Broad Range assay kit, and the A260/280 value was checked with a Nanodrop spectrophotometer. Samples were stored at $-80^{\circ} \mathrm{C}$ prior to being shipped to the sequencing center. Illumina sequencing was performed at the State University of New York at Buffalo Genomics and Bioinformatics Core Facility.

For PacBio sequencing, we had to pool DNA from multiple individuals to obtain sufficient quantities of DNA for library preparation. We performed separate DNA extractions from three individuals of each sex as above. Then, we pooled the DNA from the three individuals of each sex and concentrated it using Ampure XP beads (Beckman-Coulter). Briefly, we washed the beads three times in molecular biology grade water, once in Qiagen buffer EB, and finally resuspended the beads in their original buffer. We then added equal volumes of Ampure XP suspension to the DNA samples, mixed them on a shaker for 15 minutes, placed the tubes on a 
magnetic bead separator, and removed the supernatant. We washed the beads twice with 1.5 $\mathrm{mL}$ of $70 \%$ ethanol, and finally eluted the DNA samples in $30 \mu \mathrm{L}$ of Qiagen buffer EB.

251 Sequencing libraries were prepared and sequenced at the University of Delaware Sequencing \& Genotyping Center on a PacBio RSII.

We also supplemented our PacBio dataset with Oxford Nanopore sequencing data. We isolated DNA from a single $T$. rathkei female and two separate males using a Qiagen DNEasy Kit as described above. We then performed sequencing on Oxford Nanopore Minion flowcells (R9.4) with the Rapid Sequencing Kit (SQK-RAD004) following the manufacturer's instructions.

\section{Genome assembly}

260

We performed a hybrid assembly combining the short- and long-read sequence data, closely mirroring the bioinformatics pipeline used to generate previously published isopod genome assemblies (Chebbi et al., 2019; Becking et al., 2019). We first removed adapters and trimmed the Illumina sequencing data using Trimmomatic v. 0.36 (Bolger et al., 2014); we removed leading and trailing bases, as well as internal windows of at least $4 \mathrm{bp}$, with a mean quality score of 5 or lower, and discarded any reads shorter than 36 bp after trimming. We then used SparseAssembler (Ye et al., 2012) to assemble the cleaned Illumina data from sample Mpool, This dataset was chosen because it had decent coverage $(\sim 38 \mathrm{x})$, was generated from a PCRfree sequencing library, and came from male samples (so that $Y$ chromosome sequences would be present); additional male sequencing samples were excluded from this initial assembly to minimize the number of sequence polymorphisms that would be present in the data with additional samples. This first assembly was performed using two different kmer sizes ( $k=51$ and

$273 \mathrm{k}=61$ ). After performing preliminary quality checks using Quast (Gurevich et al., 2013), we

274 decided to proceed with the $k=61$ assembly, which had the longer total length and N50 
275 (Supplementary Table 2). However, because we suspected the genome might still contain high 276 levels of heterozygosity despite two generations of inbreeding, we used Redundans (Pryszcz 277 and Gabaldón, 2016) to remove putative allelic contigs from the Illumina-only assembly; we set 278 identity and overlap thresholds of $95 \%$.

Prior to performing hybrid assembly, we used the short reads to correct sequencing errors in the long reads using FMLRC (Wang et al., 2018) with the default settings, except requiring a minimum count of 3 to consider a path (-m 3). PacBio and Oxford Nanopore reads derived from female isopods were corrected using Illumina sample Fpool, while long reads from male samples were corrected using sample Mpool.

285

We next performed hybrid assembly using DBG2OLC (Ye et al., 2016), which accepts a shortread assembly (rather than raw short-read sequence data) and long-read sequence data (in this case, our combined PacBio and Oxford Nanopore reads) as input. We tested out a range of different parameter values: from the Redundans-filtered assembly, we first removed contigs less than $100 \mathrm{bp}$ or $200 \mathrm{bp}$; we tested kmer sizes of 17 and 19; for the kmer coverage threshold, we tried values of 2 and 5; and for the minimum overlap, we tried values of 10 and 30 . We used an adaptive threshold of 0.01 . These assemblies ranged in size from $\sim 5.2 \mathrm{~Gb}$ to $8.5 \mathrm{~Gb}$; we selected three assemblies across the range of total sizes for further processing.

We next corrected errors in these assemblies, caused by the relatively high error rates in longread sequence data. In the standard DBG2OLC pipeline, the resulting contigs are corrected using the contigs from the short-read assembly and from the long reads using Sparc (Ye and Ma, 2016); however, in our initial attempts, large portions of the assemblies went uncorrected, perhaps because we had relatively low-coverage long-read data. Therefore, instead we performed three rounds of error correction using Pilon (Walker et al., 2014), by mapping the 
trimmed Illumina sequence reads to each assembly using bbmap (first two rounds; (Bushnell et al., 2017)) and bwa mem (third round; with the parameters -A 1 -B 1 -O 1 -E 1 -k 11 -W 20 -d 0.5 -L 6 for mapping to an error-prone assembly; (Li, 2013)).

Finally, we assessed the quality of each of the three candidate assemblies using BUSCO v.3.0.2 (Simão et al., 2015), with the arthropod reference gene set, and selected the assembly with the greatest number of BUSCO reference genes present for further analysis.

To remove contaminants from the final assembly, we generated blob plots using Blobtools v.1.0 (Laetsch and Blaxter, 2017). To accomplish this, we BLASTed all contigs against the NCBI nucleotide (nt) database using megablast (Morgulis et al., 2008) and against Uniprot reference proteomes using diamond blastx (Buchfink et al., 2015). We then removed any contigs that were identified as coming from plants, fungi, viruses, or bacteria, except for those matching Wolbachia.

\section{$\underline{\text { Genome annotation }}$}

We used RepeatModeler v.1.0.10, which uses RECON (Bao and Eddy, 2002), RepeatScout (Price et al., 2005), and Tandem Repeat Finder (Benson, 1999), to construct a custom repeat library for T. rathkei. Because we were unable to run RepeatModeler successfully using the full assembly, we ran RepeatModeler on a random subset $40 \%$ of the contigs; this should still successfully identify most repetitive elements in the genome as long as all repeat families are well represented in the subset. We then masked the assembly using RepeatMasker 4.0.7, and used the data generated by RepeatMasker on divergence between each individual repeat and the consensus sequence for that family to examine the history of transposable element activity in T. rathkei (Tarailo-Graovac and Chen, 2009). 
328 We annotated coding sequences using the MAKER pipeline (Cantarel et al., 2008). We initially ran MAKER v2.31.9 using assembled transcript sequences (est2genome=1) from previously available data from one wild-caught male and one wild-caught female $T$. rathkei from the same population (Becking et al., 2017). This transcriptome was generated by assembling the male and female samples separately, each with two assemblers, Trinity v2.8.4 (Grabherr et al., 2011) and TransLiG v1.3 (Liu et al., 2019), and then merging and filtering the transcriptome assemblies with EvidentialGene v4 (Gilbert, 2019).This initial annotation also incorporated protein alignments against Uniprot-Swissprot (version March 2020), and the resulting output was used to train SNAP (Korf, 2004). To train AUGUSTUS (Stanke et al., 2006) we used the output from the BUSCO quality assessment described earlier. We then completed a final round of MAKER using the trained gene models, retaining the transcript and protein alignments from earlier as evidence.

\section{Development of sex-linked PCR markers}

We used multiple approaches to develop male-specific, putatively Y-linked PCR markers. Initial attempts to perform a hybrid Illumina-PacBio genomic assembly with only male samples and then identify contigs with zero coverage in females were unsuccessful. We therefore developed a complementary approach by looking for male-specific k-mers using just the raw Illumina 347 sequencing reads. We chose a value of $k=21$ because it should be large enough that most $k-$ mers will not occur more than once in the genome sequence, yet small enough to minimize the impact of sequencing errors (Vurture et_al..2 2017). We used kmc v.3.1.0 (Kokot et al., 2017) to count all the canonical 21-mers in each of the Illumina sequencing datasets (in other words, each 21-mer and its reverse complement were considered to be the same k-mer during counting). We then searched for k-mers that occurred at least 8 times in the Mpool Illumina 
sequencing dataset and a total of at least 3 times combined across the lower coverage M2, M5, M6, and wildM samples, but which were completely absent from all female samples. We then extracted all Illumina sequence reads containing these candidate male-specific k-mers using mirabait v.4.0.2 (Chevreux et al., 1999), and assembled them using Spades v.3.11.1 (Bankevich et al., 2012). We also performed a reciprocal analysis looking for female-specific k-mers (which are not expected in an $\mathrm{XX} / \mathrm{XY}$ system), searching for k-mers that occurred at least 8 times in the Fpool Illumina sequencing dataset, a total of at least 3 times combined across the F3, F4, and wildF samples, but which were completely absent from all male samples.

To test male-specificity of these contigs, we used PCR. We developed PCR primers for a subset of candidate male-specific contigs. To identify the best candidates, we first mapped raw sequencing reads from all male and female Illumina samples to the full genome sequence plus the candidate male-specific contigs, and identified contigs that had coverage in male samples but not female samples; we also avoided contigs that showed evidence of containing repeat elements, after BLAST searches against the whole genome assembly. We designed primers using PRIMER3 (Untergasser et al., 2012, p. 3). In these PCRs, primers were initially screened using template DNA from two male samples and two female samples; primers that showed evidence of sex specificity after this first PCR were re-tested using a larger number of samples. PCR primers were initially tested using a cycle of $98^{\circ} \mathrm{C}$ for 3 minutes, followed by 40 cycles of $98^{\circ} \mathrm{C}$ for $15 \mathrm{~s}, 50^{\circ} \mathrm{C}$ for $35 \mathrm{~s}$, and $68^{\circ} \mathrm{C}$ for $60 \mathrm{~s}$; this was followed by a final extension step of $68^{\circ} \mathrm{C}$ for 10 minutes. For samples that did not amplify under this program, a gradient PCR was run to determine optimal annealing temperature. All PCRs were performed using the same recipe and reaction conditions as the Wolbachia PCRs described above.

We also identified open reading frames (ORFs) in these candidate male-specific contigs using Transdecoder v.4.0.0 (Haas and Papanicolaou, 2016), and annotated the ORFs using Trinotate 
v.3.1.1 (Bryant et al., 2017). Subsequently, we designed additional primers targeting one of the candidate ORFs (F: 5'-ATTCTTGACTCTCCCCACGA-3'; R: 5'TCTCCAACTACGATTTCGTTAATT-3').

\section{Results}

\section{No Wolbachia and balanced sex ratios in T. rathkei}

Among the 100+ individuals captured and tested between 2015 and 2017, no T. rathkei from RCFS conclusively tested positive for Wolbachia. This was not due to inadequate testing protocols; for instance, a captive population of Porcellio laevis housed in our lab shows nearly a $100 \%$ infection rate using the same methods (not shown). Approximately $150 T$. rathkei broods were raised in the lab from either mated, wild-caught females or first-generation crosses. The mean and median brood sizes of this species in our lab were 27.1 and 22.5 offspring, respectively, and the vast majority of these broods had a balanced sex ratio (Supplementary Table 3). Thus, the prevalence of Wolbachia and other sex ratio distorters is at most very low in this population of $T$. rathkei. In addition, some wild-caught females produced broods even after several months to a year in isolation in the lab (Supplementary Table 3), confirming that this species is capable of long-term sperm storage.

\section{Crossing sex-reversed individuals indicates an $X Y$ sex determination system}

Five juveniles implanted with androgenic glands survived to mature into males; they were crossed with virgin females from families with normal sex ratios. Each putative neo-male was paired with 2 to 3 females, and each female produced 1-3 broods of offspring. Two of these males sired broods with balanced sex ratios (not significantly different from the null hypothesis 
405

of a 1:1 ratio of males to females; Table 1). These males were likely individuals that would have developed into males even without the AG implantation, but were initially mis-identified as juvenile females probably due to incomplete sexual differentiation at that early stage. Thus, these crosses are uninformative with respect to the sex determination system. A similar rate of "failed" crosses was observed in a recent study following identical protocols in other isopod species, including species without any evidence of sex chromosome polymorphism, suggesting that sexing juveniles for $A G$ implantation at these early stages is difficult due to the possibility of incomplete sexual differentiation (Becking et al., 2017). Three other males produced only female offspring, consistent with an $X X / X Y$ system ( $X X$ neo-male $\times X X$ female yields all $X X$ and therefore $100 \%$ female offspring) but not a ZZ/ZW system (ZW neo-male $\times$ ZW female expected to produce $1 / 4 \mathrm{ZZ}, 1 / 2 \mathrm{ZW}$, and $1 / 4 \mathrm{WW}$ offspring, thus $75 \%$ female or $66.7 \%$ female depending on whether WW genotypes are viable; Table 1).

\section{$\underline{\text { Genome assembly }}$}

All sequencing data have been deposited in the NCBI SRA under the project accession number PRJNA633105 (sequencing runs are SRR11797353-SRR11797365, SRR4000573, and SRR4000567); the draft assembly has been deposited under accession number GCA_015478945.1. The draft genome assembly of $T$. rathkei is approximately $5.2 \mathrm{~Gb}$ in total length. The genome is highly repetitive, consisting of approximately $70 \%$ repetitive elements. Transposable elements constitute the largest repeat category, with LINEs, followed by DNA elements and LTRs, being the most represented (Figure 1). All repeat families seem to have a single divergence peak of around 7-10\% (Figure 1).

Despite its large size, the draft assembly is likely only partially complete, with $\sim 25 \%$ of arthropod BUSCO genes missing (Table 2). For an independent assessment of assembly completeness, 
we also estimated the proportion of transcripts from the previously available transcriptome

432 dataset that were present in the assembly. There were 15,805 transcripts assembled from that 433 previously available transcriptome whose best hits in blastn searches against the NCBI nt 434 database and diamond blastx searches against Uniprot-Swissprot were from other arthropods 435 (and thus were unlikely to come from bacterial contaminants); of those, only $53 \%$ had nearly 436 full-length matches in the genome ( $\geq 90 \%$ of the transcript length at $\geq 90 \%$ sequence identity), 437 suggesting some missing data and/or remaining uncorrected sequencing errors in the draft 438 assembly as well. At a more relaxed cutoff of at least $50 \%$ of the transcript having BLAST hits in 439 the genome at $\geq 90 \%$ sequence identity, then $91 \%$ of transcripts appear to be represented 440 somewhere in the assembly, suggesting that most transcripts are indeed present in the assembly but may be partial fragments rather than whole gene sequences.

443 We screened the T. rathkei genome for Wolbachia nuclear insertions by BLASTing the 444 assembled contigs against a collection of Wolbachia genome sequences, and then BLASTing the matching regions against all representative bacterial genomes from RefSeq to rule out false positives. After this filtering step, we were left with 1,010 high confidence matches (best BLAST 447 hit in a Wolbachia genome, e-value $<1 \times 10^{-6}$ ) spread across 719 contigs, with a total length of $448 \sim 350 \mathrm{~kb}$ for the matching sequences (Supplementary Table 4), much smaller than a typical full 449 Wolbachia genome of about $1-1.6 \mathrm{Mb}$ on average (Sun et al., 2001). These may represent 450 independent small insertions into the host genome, or one or more larger insertions that were 451 subsequently broken up by mobile elements or other genomic rearrangements, or which 452 assembled into separate contigs due to insufficient data. These likely horizontally acquired 453 sequences were closely related to Wolbachia strain wCon from the isopod Cylisticus convexus 454 (Badawi et al., 2018), the feminizing Wolbachia strain wVulC, and the $f$ element of $A$. vulgare 455 (Leclercq et al., 2016) (Figure 2). 
We identified $\sim 6.04 \times 10^{6} 21$-mers as potentially male-specific, suggesting there is a minimum of $6 \mathrm{Mb}$ of male-specific sequence content in the genome. However, when we isolated the raw Illumina sequencing reads containing those 21-mers and assembled them, we obtained $89.4 \mathrm{Mb}$ of assembled sequences, suggesting the male-specific region may be as large as $\sim 90 \mathrm{Mb}$, but still shares significant similarity with the $\mathrm{X}$ chromosome. Even if up to $90 \mathrm{Mb}$ of sequence is partially sex-linked, this represents just $1.7 \%$ of the genome.

As a negative control, we also screened for k-mers specific to females. We identified $9.11 \times 10^{5}$ female-specific 21-mers using the same criteria as our analysis for male-specific k-mers, or approximately $15 \%$ the number of male-specific k-mers. The observation that there are far more male-specific 21-mers than there are female-specific 21-mers in our dataset suggests that most of these are from real male-specific genomic sequences.

Of the initial 16 candidate Y-linked PCR markers designed from anonymous sequences, none showed the expected pattern of male-specific amplification in our early tests (Supplementary Table 5). This may be due to (1) the highly repetitive nature of the $T$. rathkei genome, despite our best efforts to target primers to non-repetitive sequences, or (2) low divergence between Xand Y-linked copies.

Because the candidate male-specific contigs were assembled from Illumina data only and thus short and fragmented, we were unable to screen them for annotated candidate sex-determining genes using the typical MAKER pipeline. However, we were able to identify open reading frames (ORFs) and annotate them like transcripts using Trinotate (Bryant et al., 2017). Three contigs in the male-specific assembly showed homology to the androgenic gland hormone 
$483(\mathrm{AGH})$ gene upon annotation, suggesting there may be a Y-linked duplication of the AGH gene.

484 Therefore, we designed PCR primers specifically targeting one of the Y-linked AGH-like sequences (AGHY1 on NODE_44048_length_535, see Methods for primer sequences; same PCR cycling conditions as the other candidate sex-specific primers). These primers resulted in a PCR product of the expected size (195 bp) in all male samples screened $(7 / 7)$, but not in any of the female samples (0/7), all of which were unrelated wild-caught individuals, confirming the male-specificity of this AGH allele.

490

This AGH sequence could be either a male-specific duplication of the AGH gene, or a Y-linked allele that has diverged from an X-linked copy (in other words, gametologs). To distinguish between these possibilities, we examined the sequencing depth of these genes and of other putatively single-copy genes (identified in the BUSCO analysis) in male and female Illumina sequencing data. If the male-specific AGH sequence is a gametolog of an X-linked sequence, we would expect the total sequencing depth of all AGH sequences (putative autosomal and putative $\mathrm{Y}$-linked) to be the same in both the pooled male and pooled female samples, with the female sample having a higher average sequencing depth for the putative X-linked AGH sequences (since they would be homozygous for the X-linked gametologs, while males would be hemizygous for the X-linked gametologs). If, on the other hand, the male-specific AGH sequences are $\mathrm{Y}$-linked duplicates, and not allelic to the other AGH sequences in our assembly, we would expect the shared autosomal AGH sequences to have similar sequencing depth in

503 both male and female samples, and the combined sequencing depth of all AGH sequences 504 (putative autosomal and putative Y-linked) would be higher in the male sample. Our results were consistent with the latter scenario, suggesting these are Y-linked duplicates rather than gametologs (Figure 3). Note that sequencing depth of AGHY1 and AGHY2, though much lower 507 in the female sample than in the male sample, is still non-zero in the female sample, probably 
because of ambiguously mapped reads due to high similarity between the $\mathrm{Y}$-linked and autosomal copies.

Because the male-specific AGH sequences were found only in our Illumina data, we were unable to assemble them into long contigs, even after repeated attempts to assemble them individually with different assemblers and parameter values (not shown); all these contigs were $\sim 600 \mathrm{bp}$ or less in length. Thus we are unable to determine whether these are complete duplicates of the whole gene, or fragments. Nevertheless, a phylogenetic analysis suggests that one of the Y-linked duplicates is a copy of the other, rather than an independent duplication of an autosomal copy, and based on branch lengths they are as divergent from one another as AGH orthologs in different species (Figure 5). In addition, these Y-linked copies seem to lack an intron that is present in the autosomal copies (Figure 4), suggesting they may have originated via retrotransposition. Note that $A G H Y 1$ and $A G H Y 2$ seem to be duplicates of the same region of the AGH gene (Figure 4), suggesting they are indeed two duplicates (the fact that both copies have appreciable sequencing depth in the pooled male sample, and are $\sim 20 \%$ divergent at the DNA sequence level, suggests that these are real duplicates and not assembly artifacts; moreover, they cannot be allelic sequences because all the males in the Illumina samples that they were assembled from were siblings, carrying the same $\mathrm{Y}$ chromosome). AGHY3, on the other hand, covers a different region of the AGH gene (Figure 4), so AGHY3 may not be an additional third duplicate, but may instead be a part of AGHY1 or AGHY2 that just assembled into a separate contig.

We also find some evidence of additional autosomal duplicates of the androgenic gland hormone (AGH) gene. Two contigs in the full assembly contained annotated transcripts with at least partial homology to the expressed transcript identified as the AGH sequence, and a third contained no annotated genes but still showed high sequence similarity to AGH in BLAST 
534 searches. However, not all of the annotated exons in the first two copies matched the expressed

535 transcript, and there were unannotated portions of the same contigs that did show sequence 536 similarity to the transcript (Figure 4). Moreover, some of the matching portions of the assembled 537 contigs had less than $90 \%$ sequence identity to the expressed transcript, and analysis of the 538 sequencing depth of these regions reveals that one has very low coverage, suggesting it may 539 be an assembly artifact (see below). Thus, we cannot rule out the possibility that some of these 540 possible autosomal duplicates represent assembly and/or annotation artifacts. If they are real, 541 these autosomal duplicates appear to be specific to Trachelipus, occurring after its divergence 542 from Porcellio (Figure 5), but they may still be nonfunctional.

\section{Discussion}

We have shown that, at least in our upstate New York population, sex determination in the 547 terrestrial isopod $T$. rathkei is based on an XX/XY sex chromosome system. Two independent 548 lines of evidence support this finding: first, crosses between females and sex-reversed neomales yielded all female offspring (Table 1), consistent with an XX/XY system but not a ZZ/ZW system (Becking et al., 2017); second, we have identified PCR primers that only amplify a product in male samples, indicating the presence of a male-specific genomic region, i.e., a $Y$ chromosome.

554 Our findings run counter to a previously published study showing evidence of female 555 heterogamety in this species based on cytogenetics; in that study, female germ cells contained one set of unpaired chromosomes (presumably, the Z and W sex chromosomes), while male germ cells did not (Mittal and Pahwa, 1980). There are multiple possible explanations for this

558 contradiction. First, it is possible that the previous study incorrectly identified the species of 559 study specimens, as no information on identification is given in the paper; however, T. rathkei is 
relatively easy to distinguish from other cosmopolitan terrestrial isopod species by its five pairs of pleopodal "lungs" (most superficially similar species such as Porcellio scaber have only two pairs; (Hatchett, 1947; Shultz, 2018)). In addition, that study was published before feminizing Wolbachia was widely recognized in terrestrial isopods. It is therefore theoretically possible that the females used in that study carried an XY genotype but were feminized by Wolbachia, while the males in that study might have carried a YY genotype, perhaps resulting from a cross between an $X Y$ father and a sex-reversed $X Y$ or $Y Y$ mother which failed to transmit Wolbachia (Becking et al., 2019).

\section{Perhaps the most likely explanation is sex chromosome differences between populations.} Indeed, this would not be unprecedented, as sex determination in terrestrial isopods is thought to evolve rapidly (Rigaud et al., 1997; Cordaux et al., 2011; Becking et al., 2017), and withinspecies sex chromosome polymorphisms are documented in a few other species. For instance, two subspecies of Porcellio dilatatus, $P$. dilatatus dilatatus and $P$. dilatatus petiti have $\mathrm{XX} / \mathrm{XY}$ and ZZ/ZW systems, respectively (Juchault and Legrand, 1964; Legrand et al., 1974; Becking et al., 2017). In addition, multiple sex determining elements segregate in populations of the common pillbug $A$. vulgare (Juchault et al., 1992), including a novel W chromosome that resulted from the integration of an almost entire Wolbachia genome into the host genome (Leclercq et al., 2016). Outside terrestrial isopods, sex chromosome polymorphisms are also documented in a range of other arthropods and vertebrates (Orzack et al., 1980; Franco et al., 1982; Ogata et al., 2008; Rodrigues et al., 2013). T. rathkei is probably non-native in North America where this study was conducted (Jass and Klausmeier, 2000), as well as perhaps in India where the prior study on cytogenetics was done (Mittal and Pahwa, 1980). Given its cosmopolitan distribution, and the fact that other terrestrial isopods have moderate to high levels of genetic diversity (Romiguier et al., 2014), it might not be especially surprising for T. rathkei to harbor multiple polymorphic sex-determining loci. Nevertheless, the XX/XY system seems to be 
fixed, or at least the majority, in our population: multiple segregating sex-determining factors within a single populations usually result in sex-biased broods (Denholm 느는

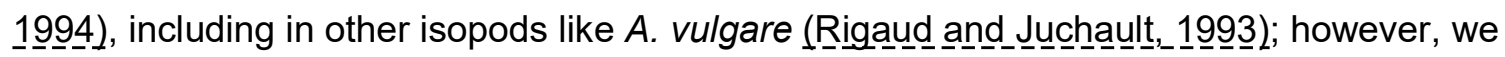
observed only a few (out of 131) sex-biased broods from wild-caught gravid females or labreared females outside of our sex reversal experiments, and never observed any single-sex broods. Moreover, the male-specific primer pair we designed amplified successfully in all tested wild-caught males, and none of the wild-caught females, albeit with modest sample sizes. Hopefully future follow-up work can further characterize geographic variation in sex determination in this species.

Regardless of whether or not sex determination is polymorphic in our population of T. rathkei, we propose that the ZZ/ZW sex chromosomes in this species are more likely to be ancestral, and that the $\mathrm{XX} / \mathrm{XY}$ system is derived. First, $T$. rathkei is nested within a clade that mostly consists of ZZ/ZW species (Becking et al., 2017). Moreover, the previous study finding a ZZ/ZW system in $T$. rathkei was based on the presence of heteromorphic sex chromosomes in female meiotic spreads (Mittal and Pahwa ${ }_{2}$ 1980), suggesting that the $\mathrm{Z}$ and $\mathrm{W}$ have been diverging long enough to be cytogenetically distinguishable, in contrast to other isopods examined so far showing homomorphic sex chromosomes (Rigaud et al..2 1997). In addition, the putative malespecific region of the $T$. rathkei genome does not contain any genes that are essential for male reproduction or spermatogenesis, since phenotypic males with an XX genotype still sired offspring in our sex-reversal experiments, and the male-specific region is only a small portion of the total genome, similar to other terrestrial isopods examined so far (Chebbi et al., 2019; Becking et al., 2019). This male-specific region is probably at least $6 \mathrm{Mb}$ and has an upper size limit of around $90 \mathrm{Mb}$, but these estimates include sequences that retain high similarity to Xlinked copies; indeed, most of the candidate male-specific primers we tested failed to show sexspecific amplification patterns. These observations suggest that this $Y$ chromosome may be 
613 Given that we found genomic evidence of a past association with Wolbachia in this species and 614 that infection by Wolbachia has been found in other T. rathkei populations (Cordaux et al.,

evolutionarily young, since it has not had time to accumulate major differences from the $\mathrm{X}$. 2012), this scenario is consistent with the hypothesis that transitions in sex determination mechanisms may be triggered by Wolbachia and other endosymbionts that manipulate host reproduction (Rigaud et al., 1997; Cordaux et al., 2011). If other populations of T. rathkei with different sex determination mechanisms can be identified, it may be possible to leverage this system to further study the mechanisms and selective forces influencing transitions in sex determination mechanisms. In addition, studies of sex determination in a phylogenetic context involving other members of the family Trachelipodidae would shed further light on the origins of the $X$ and $Y$ chromosomes in T. rathkei.

The draft genome assembly of $T$. rathkei is especially large, at around $5.2 \mathrm{~Gb}$, with approximately $29 \%$ GC content. The actual genome is likely to be even larger, given that $\sim 25 \%$ of the BUSCO arthropod orthologs were missing in our assembly. By comparison, genomes of pillbugs in the genus Armadillidium tend to be smaller at around 1.2 - $2 \mathrm{~Gb}$ in size (Chebbi et al., 2019; Becking et al., 2019), but other terrestrial isopods have genomes ranging to over $8 \mathrm{~Gb}$ (Gregory, 2020), and other crustacean relatives such as amphipods also have large genomes (Rees et al., 2007; Rivarola-Duarte et al., 2014; Kao et al., 2016), so T. rathkei is not out of the ordinary for this group.

The T. rathkei genome contains a large proportion of repetitive elements, in particular transposable elements (Figure 1). The most common transposable element families are LINEs, DNA elements, and LTRs, similar to A. vulgare and A. nasatum (Chebbi et al., 2019; Becking et al., 2019, 2020). The distribution of divergence values, with a single mode around $7-10 \%$ divergence (Figure 1), suggests that most repeat families expanded around the same time as 
previously shown in A. vulgare and A. nasatum; however, unlike in A. vulgare, T. rathkei shows no evidence of a second more recent burst in DNA element activity. Simple repeats also comprise a substantial portion of the genome; even manually looking through the assembled contigs reveals a high abundance of $(\mathrm{TA}) \mathrm{x}$ repeats. It would be interesting to examine the repeat content of the male-specific portion of the genome. Unfortunately, however, we were only able to recover male-specific sequences from the short-read Illumina data, and this portion of the genome assembly is highly fragmented, precluding more detailed analysis. Hopefully, additional long-read sequencing data will allow us to examine transposable element dynamics in this area in the future.

We found many contigs with high similarity to the Wolbachia genome (Supplementary Table 4), even though we were unable to detect current Wolbachia infections in our population using PCR. This is not surprising given that horizontal transfers of Wolbachia DNA into host genomes is common (Dunning Hotopp ${ }_{2}$ 2011), and that Wolbachia is relatively common in terrestrial isopods and arthropods in general (Cordaux et al., 2012; Pascar and Chandler, 2018; Medina et al., 2019) and has been found in other populations of $T$. rathkei. These Wolbachia insertions are closely related to other Wolbachia strains from isopods, including feminizing strains (Cordaux et al 2004, Leclercq et al 2016). This suggests that T. rathkei may have been infected with a feminizing Wolbachia strain in the past, even though no firm conclusion can be drawn solely from phylogenetic evidence. If so, it is conceivable that Wolbachia may have been involved in the sex chromosome turnover we characterized in $T$. rathkei, as previously hypothesized (Rigaud et al 1997, Cordaux and Gilbert 2017).

Male differentiation in terrestrial isopods is controlled by the androgenic gland hormone, AGH. AGH is a peptide hormone similar in structure to insulin, and is secreted by the androgenic gland (Martin et al., 1999). AGH expression is sufficient to transform juvenile female isopods 
664

665

666

667

668

669

670

671

672

673

674

675

676

677

678

679

680

681

682

683

684

685

686

687

688

689

into fertile males (Martin et al., 1999). Presumably, in wild-type males, the primary sexdetermining signal triggers the differentiation of the androgenic glands during development, which then secretes AGH. Interestingly, the draft genome of T. rathkei contains multiple AGHlike sequences, unlike $A$. vulgare, which has a single copy (Chebbi et al 2019). While some of these may be assembly artifacts, there is evidence of at least two partial Y-linked sequences (assembled from Illumina sequencing reads containing male-specific k-mers), of which one was confirmed by PCR to be male-specific. These duplications seem to be specific to $T$. rathkei (Figure 5), though other members of the genus Trachelipus or the family Trachelipodidae have yet to be examined. Consistent with this, a past study found no evidence of any expressed AGH duplications in other terrestrial isopod species except Porcellio gallicus (Cerveau et al., 2014).

In many other species, novel sex chromosomes have arisen via duplication of a sex-determining gene. For instance, duplicates of the vertebrate gene Dmrt1 have evolved into master sexdetermining signals on the $\mathrm{W}$ and $\mathrm{Y}$ chromosomes, respectively, in the frog Xenopus laevis (Yoshimoto et al., 2008) and the medaka Oryzias latipes (Matsuda et al., 2002, 2007; Nanda et al., 2002), while a Y-linked duplicate of the anti-Müllerian hormone gene is a candidate master sex-determining gene in the teleost fish Odontesthes hatcheri (Hattori et al., 2012). The presence of Y-linked AGH copies in T. rathkei, and no other obvious open reading frames homologous to known sex determination or sex differentiation genes, makes these genes obvious candidates for the master male-determining signal in T. rathkei. Sex-specific genomic regions like the $\mathrm{Y}$ and $\mathrm{W}$ chromosomes are also expected to acquire sexually antagonistic alleles (van Doorn and Kirkpatrick, 2007, 2010; Charlesworth, 2017).Thus, if functional these duplicates might instead provide male fitness benefits rather than serving as a master male sexdetermining gene. Unfortunately, we were unable to assemble full copies of these Y-linked AGH homologs because they only showed up in our Illumina data, not in our low-coverage long read data. Future deep sequencing using long reads should further clarify the molecular evolution of 
690 these genes. In addition, expression studies should determine which of these genes are 691 expressed, in what tissues, and at what stages.

692

693 We have shown that the terrestrial isopod T. rathkei uses an XX/XY sex chromosome system,

694 at least in upstate New York, in contrast to a past cytogenetic study suggesting a ZZ/ZW

695 mechanism (Mittal and Pahwa, 1980). In line with this, whole-genome sequencing and follow-up

696 PCRs demonstrate the existence of male-specific, Y-linked copies of the androgenic gland

697 hormone gene in this species. These findings highlight the role of gene duplication in the

698 evolution of sex chromosomes and they further establish terrestrial isopods as models to study

699 the evolution of sex determination systems and the mechanisms underlying their transitions.

700 


\section{Acknowledgments}

702

703 We thank the editor and three anonymous reviewers for constructive comments on earlier drafts

704 of this manuscript. We also appreciate computing time and assistance provided by the National

705 Center for Genome Analysis Support at Indiana University, especially Tom Doak and Sheri

706 Sanders. This research was funded by National Science Foundation grant NSF-DEB 1453298

707 to $\mathrm{CHC}$. It was also supported in part by Lilly Endowment, Inc., through its support for the

708 Indiana University Pervasive Technology Institute. This research is based upon work supported

709 by the National Science Foundation under Grant Nos. DBI-1062432 2011, ABI-1458641 2015 ,

710 and $\mathrm{ABI}-17599062018$ to Indiana University. Any opinions, findings, and conclusions or

711 recommendations expressed in this material are those of the authors and do not necessarily

712 reflect the views of the National Science Foundation, the National Center for Genome Analysis

713 Support, or Indiana University.

714

715 
Table 1. Sex ratios from crosses between putative neo-males (juvenile females implanted with an androgenic gland) and females. The last three columns give the results of chi-square tests

718 testing whether the observed sex ratios (pooling the results for each male) are significantly

719 different from predicted ratios of 1 female: 1 male, 2 female : 1 male, and 3 female : 1 male.

\begin{tabular}{|c|c|c|c|c|c|c|}
\hline Neo-male & Female & $\begin{array}{l}\text { Number of } \\
\text { female } \\
\text { offspring }\end{array}$ & $\begin{array}{l}\text { Number of } \\
\text { male } \\
\text { offspring }\end{array}$ & $50 F: 50 M$ & $66 \mathrm{~F}: 33 \mathrm{M}$ & $75 F: 25 M$ \\
\hline \multirow[t]{3}{*}{ D-4-7 } & 1 & 23 & 0 & \multirow{3}{*}{$\begin{array}{l}x^{2}=44 \\
p=3.28 x \\
10^{-11}\end{array}$} & \multirow{3}{*}{$\begin{array}{l}x^{2}=22 \\
p=2.73 x \\
10^{-6}\end{array}$} & \multirow{3}{*}{$\begin{array}{l}x^{2}=14.7 \\
p=1.28 x \\
10^{-4}\end{array}$} \\
\hline & 2 & 14 & 0 & & & \\
\hline & 3 & 7 & 0 & & & \\
\hline \multirow[t]{3}{*}{ G-4-22 } & 1 & 16 & 8 & \multirow{3}{*}{$\begin{array}{l}x^{2}=1.49 \\
p=0.22\end{array}$} & \multirow{3}{*}{$\begin{array}{l}x^{2}=3.56 \\
p=0.059\end{array}$} & \multirow{3}{*}{$\begin{array}{l}x^{2}=14.3 \\
p=1.54 x \\
10^{-4}\end{array}$} \\
\hline & 2 & 17 & 14 & & & \\
\hline & 3 & 13 & 13 & & & \\
\hline \multirow[t]{2}{*}{$F-4-9$} & 1 & 36 & 0 & \multirow{2}{*}{$\begin{array}{l}x^{2}=59 \\
p=1.58 x \\
10^{-14}\end{array}$} & \multirow{2}{*}{$\begin{array}{l}x^{2}=29.5 \\
p=5.59 x \\
10^{-8}\end{array}$} & \multirow{2}{*}{$\begin{array}{l}x^{2}=19.7 \\
p=9.22 \mathrm{x} \\
10^{-6}\end{array}$} \\
\hline & 2 & 23 & 0 & & & \\
\hline \multirow[t]{2}{*}{$10-8$} & 1 & 24 & 35 & \multirow{2}{*}{$\begin{array}{l}x^{2}=0.87 \\
p=0.35\end{array}$} & \multirow{2}{*}{$\begin{array}{l}x^{2}=26.6 \\
p=2.50 x \\
10^{-7}\end{array}$} & \multirow{2}{*}{$\begin{array}{l}x^{2}=62.2 \\
p=3.17 \mathrm{x} \\
10^{-15}\end{array}$} \\
\hline & 2 & 40 & 40 & & & \\
\hline \multirow[t]{2}{*}{ AGS169-2 } & 1 & 45 & 0 & \multirow{2}{*}{$\begin{array}{l}x^{2}=82 \\
p=1.36 x \\
10^{-19}\end{array}$} & \multirow{2}{*}{$\begin{array}{l}x^{2}=41 \\
p=1.52 x \\
10^{-10}\end{array}$} & \multirow{2}{*}{$\begin{array}{l}x^{2}=27.3 \\
p=1.71 x \\
10^{-7}\end{array}$} \\
\hline & 2 & 37 & 0 & & & \\
\hline
\end{tabular}


723 Table 2. Assembly statistics for the T. rathkei draft genome.

\begin{tabular}{|l|l|}
\hline Total length & $5,181,251,014 \mathrm{bp}$ \\
\hline Number of contigs & 421,784 \\
\hline N50 & $39,761 \mathrm{bp}$ \\
\hline GC content & $29.0 \%$ \\
\hline Complete BUSCO genes & 533 single copy $(51.9 \%) ; 39$ duplicated $(3.7 \%)$ \\
\hline Fragmented BUSCO genes & $203(19.0 \%)$ \\
\hline Missing BUSCO genes & $271(25.4 \%)$ \\
\hline
\end{tabular}

724

725 


\section{Figure Legends}

Figure 1. Distribution of divergence levels for repetitive elements in the $T$. rathkei genome.

Figure 2. Phylogenetic tree showing the relationship of candidate horizontally transferred Wolbachia segments in the T. rathkei genome to other Wolbachia isolates. The T. rathkei Wolbachia insertion (indicated by the asterisk) is closely related to Wolbachia isolates from other isopods, and its closest relative is the wCon from Cylisticus convexus. Numbers by nodes indicate bootstrap support. Branch lengths represent average number of substitutions per site. The tree was generated by concatenating all candidate Wolbachia insertions in $T$. rathkei longer than $1,000 \mathrm{bp}$, along with the best-matching regions in the reference Wolbachia genomes (found with BLAST), aligning with MUSCLE v.3.8.31 (Edgar, 2004), filtering alignments with trimal v. 1.2rev59 (Capella-Gutiérrez et al., 2009), selecting a model using ModelTest-NG v. 0.1.6 (Darriba et al., 2020), and running the analysis in RAxML-NG v. 0.9.0 (Stamatakis, 2014) with 100 bootstrap replicates.

Figure 3. Distribution of sequencing depth for single-copy BUSCO genes in male and female Illumina sequencing datasets (M-pool and F-pool). Labeled dots indicate the sequencing depth for the different AGH copies in each sample.

Figure 4. Possible duplicates of the androgenic gland hormone gene in the $T$. rathkei genome, including male-specific duplicates (on the right). The green bars represent the sequence of the expressed AGH sequence, assembled from previously available transcriptome data. Gray bars represent contigs in the draft genome assembly, and the pink bars on contigs represent exons annotated by MAKER. Dark blue segments connecting portions of the transcript to portions of contigs represent BLAST hits; light purple connector segments represent BLAST hits in reverse 
752 orientation. The incongruence between annotated exons and BLAST matches between the 753 transcript and contigs suggests the annotation still contains some errors.

754

755 Figure 5. Phylogenetic tree showing relationships among AGH sequences from terrestrial isopods. AGH2 and AGHY3 are missing from this phylogeny because those sequences were 757 omitted because of their short length. The tree was generated using all AGH-like sequences 758 from $T$. rathkei of at least $100 \mathrm{bp}$, along with reference AGH nucleotide sequences from other 759 species, aligning them with MUSCLE v. 3.8.31 (Edgar, 2004), selecting a model using 760 ModelTest-NG v. 0.1.6 (Darriba et al., 2020), and running the analysis in RAxML-NG v. 0.9.0 761 (Stamatakis, 2014) with 100 bootstrap replicates.

762

763

764 
References

767

768 Automatic citation updates are disabled. To see the bibliography, click Refresh in the Zotero tab. 769 